\title{
Le Philosophe et le Peintre
}

\author{
Pierre Magré \\ Artiste peintre
}

Comme on le sait, Michel Henry, au cours de son cheminement de pensée, s'est toujours intéressé à l'art et plus particulièrement à l'inventeur de ce qu'il est convenu d'appeler désormais l'art abstrait. C'est en effet à travers l'œuvre de Kandinsky et de son livre Du spirituel dans l'art que Michel Henry a procédé à une analyse approfondie en remontant au Principe même de la Création. ${ }^{1}$

Après la lecture de cet ouvrage, je me suis mis en relation avec Michel Henry. Nous avons alors convenu d'une invitation à passer quelques jours dans notre campagne poitevine (dans notre habitation et notre atelier).

C'est ainsi qu'un soir, Danielle, revenant de Poitiers (Danielle Grimaldi, ma femme et mon égale en peinture), nous a trouvés tous les deux, tout bonnement assis par terre, en grande conversation au pied d'une toile posée à même le sol, tout près d'une fenêtre de l'atelier (cet emplacement avait été choisi par notre visiteur pour mieux en apprécier tous les détails).

Sur l'origine de la création artistique, l'accord fut complet et immédiat. Pour faire comprendre ma démarche qui me poussait, non plus à lancer des directions sur la surface de la toile, mais à faire venir les formes comme «facialement» du fond à la surface, je me souviens avoir eu recours à cette citation de Victor Hugo : «La poésie, c'est le fond qui remonte à la surface ». Intuition vraie mais qui demandait à être solidement fondée en raison.

On sait qu'en toute démarche, le point de départ est primordial. Quelle est la source d'inspiration du peintre? D'où vient le contenu de l'œuvre? De l'intérieur ou de l'extérieur?

Même lorsqu'il s'agit des prestigieuses séries des Nymphéas de Claude Monet, nous étions de ce même avis que ces œuvres, malgré leur très forte interprétation de la nature, demeuraient figuratives, c'est-à-dire régies par l'ordre de la nature extérieure et ne pouvaient pas conduire à l'art abstrait. Les impressionnistes, y compris le Monet des Nymphéas, allaient "sur le motif». La nature extérieure, l'impact sensoriel, était leur guide et leur mobile, leur source d'inspiration. En ce cas, quel qu'ait été le degré d'interprétation de la nature, ils en partaient et en restaient toujours plus ou moins tributaires par la suite (et heureusement pour la cohérence de leurs œuvres). Même constat pour Picasso dont la forte altération des formes ne pouvait se dispenser du monde extérieur.

${ }^{1}$ Cf. M. Henry, Voir l'invisible. Sur Kandinsky, Paris, F. Bourin, 1988. 
Nous convenions que la voie abstraite, quant à elle, trouve son origine en amont des sens extérieurs. (Proposition qui peut paraître surprenante : ainsi, le peintre, dans la première phase de son inspiration créatrice, n'aurait pas besoin de la vue.) Cette voie consiste à s'immerger dans « la nuit des sens », à remonter à l'origine de notre être où il n'y a plus alors que le surgissement de la vie. Et il n'y a rien en deçà. C'est donc une réalité incontournable et qu'on ne peut pas sub-vertir (puisqu'il n'y a rien en « dessous » ni en « deçà »). Ainsi, à chaque pulsation de notre cœur, c'est la vie qui naît et renaît en nous. Il n'y a plus désormais dans notre nuit qu'à y co-naître, selon le jeu de mot de Paul Claudel, à naître avec elle, à y adhérer en consentement à ce qui est la cause de notre être. Certes, la vie est invisible et impalpable ; la saisir, nous ne le pouvons pas; mais c'est elle qui nous saisit (si bien que si vous essayez de vous en défaire ... vous mourez ... elle est donc incontournable). Vie qui est « en nous-mêmes plus nous-mêmes que nous » (St Augustin) et qui pousse en notre être (à la fois une poussée et une croissance de notre être). Cette vie demeure notre motif, le moteur, le mobile de ce que l'on nomme l'inspiration.

Reste la fonction du peintre : agir par et avec la matière, permettre à la vie invisible de parvenir le plus pleinement possible à la visibilité. Cette démarche, c'est, en ce qui concerne le peintre : «montrer l'invisible»; en ce qui concerne le spectateur : « voir l'invisible ». C'est bien là le titre et l'objet du livre de Michel Henry pour qui l'art est «l'essence de la Vie exprimée de manière sensible », la manifestation à proprement parler spectaculaire et donc privilégiée de cette éclosion de l'essentiel invisible dans ce «monde de l'apparence ».

Cette nouvelle façon d'envisager la création, c'est celle que, dès l'âge de vingt ans, nous avons suivie et dont témoignent nos œuvres. Elles sont des illustrations vivantes des principes posés par Kandinsky (Du spirituel dans l'art, 1911), analysés par Michel Henry (Voir l'invisible, 1988) et développés par nous-mêmes (Pierre Magré et Danielle Grimaldi, Art abstrait, défense et illustration, 2011).

Un avantage des arts plastiques, c'est que, concrets, visibles, ils s'installent sereinement et durablement, non seulement dans l'espace et même dans « l'espace social » le plus commun (une peinture, une sculpture sont « meubles par nature » nous disent les juristes) mais aussi dans un présent permanent et ainsi pour chacun d'en expérimenter à loisir le contenu.

Se trouve ainsi proposé un terrain de rencontre. C'est ici en effet que le philosophe peut trouver dans le travail du peintre un atelier d'expérimentation où peut s'observer le passage du pensé au réalisé ; du pensé invisible au réalisé matériel. Un banc d'essai en quelque sorte où l'on pourra juger objectivement (mais aussi toujours sensiblement) du résultat, de « ce que ça donne » : échecs et réussites.

Demeure donc la question : cette intuition pure, sous quelle forme pourra-t-elle apparaitre sur la toile si l'on a, par principe, congédié le monde naturel : ses formes et son ordre ? Comment les formes pourront-elles se constituer en elles-mêmes ? Comment vont-elles se coordonner? Sans l'aide de la nature, en effet, comment ne 
pas piétiner dans des gribouillis abscons, dans des explosions anarchiques de formes et de couleurs, dans des affaissements ou des errements inanes ?...

Michel Henry avait en partie répondu à ces questions en analysant les fameuses propositions de Kandinsky de Point, ligne sur plan. Cependant ce peintre, cet explorateur d'une voie artistique nouvelle n'avait pu poser que quelques «lois» fondamentales de composition. Sans aucune nuance péjorative, on peut admettre qu'avec les œuvres de Kandinsky, on en est à une sorte de «primitivisme abstrait » (comme il y eut les primitifs flamands ou les primitifs italiens — dont Michel Henry était entre autres un grand amateur) : couleurs légères, fond uni ou simple, importance du trait de cerne, etc. Kandinsky dans ses écrits (et dans ses œuvres qui les confirment) pose quelques lois issues de sa pratique, lois propres à engendrer - et suffisantes à gérer - ces formes simples, souvent géométriques, plates et peu complexes (je fais bien sûr référence à la période de maturité en éludant la période transitoire lyrique où il se cherchait encore). Mais déjà, avec une grande lucidité, il note que «l'art abstrait, devenu autonome, doit subir « les lois de la nature » qui, partant du protoplasme et des cellules, est arrivé lentement à des organismes toujours plus complexes. $»^{1}$ Mais songeons à Rubens ou à Titien, et à la suite qu'ils donnèrent au «primitivisme» précédent. Nous voyons alors une orchestration beaucoup plus vaste de mouvements plus profus et variés, de formes beaucoup plus complexes qui, par leurs contrastes de valeurs, sont propulsées dans un espace plus profond et parviennent à s'imposer par une présence et une épaisseur quasiment charnelle.

Cette montée en puissance de la forme devait nécessairement s'accompagner d'une saisie beaucoup plus large des tenants et des aboutissants des modalités qui régissent les formes. Songeons à la musique : l'invention et l'accroissement de la polyphonie (invention de notre culture européenne, trésor unique au monde) a dû s'accompagner d'une mise au jour des modalités propres à la musique, de traités d'harmonie d'une extrême complexité jointe à une magnifique précision. Sans parler des instruments eux-mêmes, aussi complexes, variés et précis dans leur conception et réalisation.

Revenons à notre siècle et à la peinture. Au cours de nos discussions avec Michel Henry, le problème de l'« incarnation» de la réalité invisible à travers des formes visibles, même s'il n'était pas explicite, restait sous-jacent. Nous voyons en effet que la voie figurative s'appuie sur l'ordre du monde extérieur : la perspective qui ordonne dans l'espace, la lumière naturelle qui fait « tourner» les volumes dans ce même espace, etc. Mais le peintre qui s'abstrait de tout cela, comment établira-t-il une composition de formes qui soient en elles-mêmes « viables » et qui répondent à notre exigence d'êtres cohérents, doués de raison, laquelle en toute chose cherche une intelligibilité ?

\footnotetext{
${ }^{1}$ V. Kandinsky, Point, ligne sur plan, Paris, de Beaune, 1963, p. 91.
} 
À la vérité, en tant que peintres, nous ne nous sommes jamais posé objectivement et clairement de telles questions. Nous avons travaillé. Et c'est par la pratique que le mode même d'apparaitre de la Vie, que des «modalités» ou, si l'on veut, des « lois » plastiques se sont révélées, pour ainsi dire, sous notre main et par nos actes de praticien. En cela, aucune théorie a priori, aucune idéologie mais l'expérience et toujours le contact avec le réel, le réel de l'expérience intérieure et, inséparablement, le réel du travail de la matière. Sincérité de cœur et honnêteté déontologique. «Tête dure et cour tendre $»$.

Si bien qu'au cours d'un travail long et acharné, les formes plates se sont gonflées ; elles ont pris épaisseur et relief; elles se sont en quelque sorte faites « chair». On peut dire, d'une certaine manière, qu'elles se sont « incarnées ». Or Michel Henry, particulièrement sensible à l' " incarnation » de cette force créatrice, a rapidement saisi l'intérêt et l'aspect totalement inédit de notre démarche. Les formes ainsi créées devaient nécessairement s'adapter au milieu dans lequel elles devaient évoluer. Comme l'avait déjà affirmé Kandinsky, « la nature crée ses formes pour son but ; l'art crée ses formes pour le sien». Notre monde à trois dimensions et dans le temps devient, quand on travaille dans la réalité plastique de la surface picturale, un monde à deux dimensions et inscrit comme hors du temps. Ainsi ces modalités propres à la création artistique sont d'une importance capitale. Elles sont le mode même de la vie émergeant à la visibilité. Ce sont les « lois naturelles » de toute création artistique (à rapprocher par exemple de la loi naturelle morale). « Ces modalités, inhérentes à la Vie, sont les nécessaires et naturels régisseurs des formes. ${ }^{1}$

Une grande difficulté pour le peintre mais aussi pour le philosophe, est bien l'entrée en contact de l'expérience intérieure avec le monde matériel; le mystère du passage de l'un à l'autre; le mystère de la création, celui de toute incarnation. On peut noter ici que c'est précisément pendant notre progression dans une voie de plus grande et authentique incarnation que Michel Henry, de son côté, se dirigeait vers l'élaboration de son très bel ouvrage Incarnation. ${ }^{2}$

En ce printemps 1989, date de notre première rencontre à notre atelier à Chabournay (« Haut lieu de l'art contemporain » ainsi que l'avait nommé Michel Henry avec son humour habituel), nos œuvres se présentent déjà nanties d'une large charge de « rhétorique baroque ». Et cela a de quoi surprendre, à une époque comme la nôtre, où les arts dérivent dans les sombres vertiges d'une «culture de mort». Sans doute Michel Henry a-t-il été quelque peu surpris par certaines de nos peintures. Je l'entends encore, après qu'il eut franchi la porte de l'atelier : "Mais c'est la grande manière ! » (prononcé à l'italienne, non sans un certain enthousiasme).

La surprise fut assez vite dissipée chez notre philosophe. L'enthousiasme prit le dessus et il se lança dans une étude extrêmement approfondie des œuvres. Dès le deuxième jour, en effet, au cours d'un de nos repas, notre commensal nous annonça

\footnotetext{
${ }^{1}$ P. Magré et D. Grimaldi, Art abstrait, défense et illustration, Paris, Somogy, p. 45.

${ }^{2} \mathrm{M}$. Henry, Incarnation. Une philosophie de la chair, Paris, Seuil, 2000.
} 
avec une joie non dissimulée avoir établi le lien entre sa pensée et l'orientation de notre peinture.

Il se trouva qu'à cette époque, je projetais une exposition dans une Galerie de Poitiers et Michel Henry accepta d'en écrire le catalogue édité à cette occasion. Ce fut l'objet du premier texte, intitulé pour la circonstance «Peindre l'invisible». En réalité, ce titre a été préféré à un autre plus éloquent et plus ambitieux certes, mais qui (censure oblige!) aurait pu heurter certains esprits hostiles de nos institutions culturelles : «Une théologie de la création». Tel avait été en effet le titre initial proposé par Michel Henry. Ce ne sera que plus tard, en 1999, pour une grande exposition réalisée au Musée de Toulon, que nous reprendrons ce texte (dont on supprimera certaines parties correspondant à des œuvres non présentées à Toulon) et cette fois avec son titre exact : «Une théologie de la création ». C'est cette version qui figure dans le catalogue du Musée de Toulon.

Concernant la démarche et la réaction de Michel Henry, nous avons noté, dans un premier temps, une certaine surprise de sa part, due à l'aspect baroque de notre peinture. Or c'est précisément le mouvement propre au baroque qui a été le facteur déclenchant. En effet, le mouvement, expression de la Vie, étant présent, il suffit de se brancher sur le rythme des formes, de l'éprouver, pour en comprendre le processus.

(Par « baroque », il faut entendre l'esprit baroque dans son ensemble, celui peutêtre de la Contre Réforme dont en peinture le héros — et le héraut le plus démonstratif - a été l'étonnant - et le détonnant - chantre de la vie Pierre Paul Rubens. L'âge baroque en effet aura su conjoindre le sentiment et la raison — soit en termes artistiques : le fond et la forme — ou encore la vie dans son principe et dans ses conséquences incarnées.

Affirmation du sentiment? Oui, s'il s'agit du sentiment de la Vie et non de sentimentalité - et exprimé avec quelle fougue dans l'audace ! « Tantum potes, quantum aude! » Tant chez Vivaldi, Bach ou Haëndel que chez les architectes partout en Europe. Pensons à l'alliance du cœur et de la raison chère à Pascal. Sans oublier que la conciliation de la foi (le sentiment, le cœur) et de la raison ont permis, par nombre de savants jésuites entre autres, une considérable avancée de la pensée mathématique et scientifique.

Incontestablement, Michel Henry est un philosophe majeur de notre temps; et l'homme était en outre doué d'une sensibilité extrêmement vive et fine. J'en veux pour preuve cette anecdote : il y eut à l'Université de Poitiers, dans le courant de l'été 1992, un grand Colloque international de Philosophie de langue française dont le Président d'honneur était Michel Henry. Celui-ci nous avait suggéré de monter parallèlement une exposition de nos œuvres à intégrer dans le programme du colloque qui était cette année-là « la Vie et la Mort». À défaut du Musée de Poitiers, nous avons investi l'immense Salle des pas perdus du Palais de Justice. Danielle, pour sa part, avait exposé une suite de vingt grands tableaux : «Variations sur l'Amour ». Chaque panneau avait un titre. Lors de la visite que nous fîmes avec M. 
Henry, nous avons parcouru l'exposition, œuvre après œuvre, pendant deux heures. Il s'arrêtait à chaque panneau et disait ce qu'il ressentait et, avec une justesse d'appréciation exceptionnelle, arrivait toujours à définir le contenu et le titre de l'œuvre. Arrivé devant une peinture, il s'exclama : «Mais ... ici, c'est le Sacrement de l'Amour! » Danielle fut à ce moment assez interloquée mais heureuse cependant car Michel Henry avait vu juste ! C'était bien le titre initial de l'œuvre ! Or celui-ci avait été modifié, sur les conseils d'un entourage sans doute plus soucieux de laïcité que de transcendance à l'égard de ce lieu public, et avait été rebaptisé " Amour Sagesse »! Ainsi Michel Henry, laissant parler sa sensibilité, a spontanément trouvé le vrai sens de l'œuvre. Or ceci, ironie du sort, se passait dans la grande salle des Comtes du Poitou, là même où Jeanne d'Arc a été interrogée par les Docteurs de la Loi. Or, n'avait-on pas essayé d'abuser Jeanne en changeant le «titre» de roi et en l'attribuant par déguisement à un autre ? Par ailleurs, le roi légitime s'était réfugié à Chinon ; de même nos peintures avaient dû se « réfugier » dans la salle des Comtes du Poitou, désormais Palais de Justice, ayant été refusées par le Musée, lieu pourtant légitime pour une exposition de peintures de cette importance ( - malheur des temps !)...

Cette anecdote nous éclaire (outre l'ostracisme des institutions culturelles) sur ce fait trop souvent contesté que l'art abstrait (de même que la musique) peut signifier non seulement très fortement mais parfois aussi très précisément. Pourvu qu'on ait affaire à une œuvre authentique et à un public sensible.

Ainsi, en tout domaine, une vive intelligence est utile, voire nécessaire; mais seule, que ferait-elle ? Que ferait-elle sans une relation avec la sensibilité, avec une subjectivité radicale? Sa fonction n'est-elle pas de servir la vie en favorisant son éclosion à travers la matière dans une intelligibilité efficiente ? C'est du moins là un rapport que le philosophe peut entretenir avec l'artiste : détecter le sens le plus profond, le plus vrai, d'une création qui a pour but de révéler l'invisible, qu'il s'agisse de création humaine ou de la Création divine elle-même. La rencontre avec Michel Henry et son œuvre aura été pour nous une aide à voir plus clair dans ce phénomène de la création que l'on vit pratiquement mais plus ou moins inconsciemment, à permettre plus d'efficacité dans notre travail. Descartes avait-il tort en disant que «d'une plus grande clarté dans l'entendement s'ensuit une plus grande inclination dans la volonté » (c'est-à-dire pour nous, peintres, une plus grande efficacité dans la réalisation de l'œuvre) ?

Ainsi, d'années en années, parfois d'assez près, bien souvent de plus loin, nous avons suivi avec admiration et émotion une montée spirituelle (qui est aussi bien un approfondissement philosophique et spirituel). Se sont succédé C'est Moi la vérité (1996), Incarnation (2000) et enfin Paroles du Christ (2002).

On aura d'ailleurs pu remarquer que, dans la suite de ses ouvrages, le vocabulaire et certaines expressions ont changé. " Douleur du monde», "souffrance», "pathos » ont fait place à « la force de la vie », à son « ivresse », puis, de plus en plus 
souvent, à « l'épanouissement de la vie », « la joie » ... L'homme lui-même, acharné à débusquer sa vérité profonde et à défendre ce trésor contre toute fausse interprétation, s'est comme éclairci dans une grande "légèreté de l'être », une grande sérénité (résurgence d'une naissance en Extrême Orient?). À tel point que, sachant pourtant par son frère médecin sa fin prochaine, il n'en a rien laissé paraître. Elégance naturelle de sa personne, certes, mais sans doute aussi certitude surnaturelle.

Intelligence, sensibilité et une troisième qualité de l'homme aura été le courage. Courage pour suivre une voie personnelle de vérité, malgré le mépris et le refus d'une société en dérive. Et courage et générosité aussi pour nous aider à divulguer notre œuvre en milieu hostile. Il n'a jamais eu peur de se donner à fond jusqu'à l'épuisement, nous disait-il, pour répondre à l'appel si fort en lui-même et accomplir sa vie jusqu'au bout. Sa modestie était particulièrement touchante et sa grandeur d'âme résidait dans la dignité et le respect que lui inspirait la personne humaine, magnifique incarnation de la Vie.

Pour tout cela nous avons envers Michel Henry une grande reconnaissance; reconnaissance dans les deux sens du mot. D'une part, gratitude, d'autre part, parce que l'essentiel de sa pensée, par une sorte de communauté d'être, nous l'avons « reconnu » en nous. Au reste, l'expérience nous enseigne que nous n'apprenons rien d'essentiel que nous n'ayons déjà en nous. Toute connaissance intellectuelle est en fait une re-connaissance, ou plus exactement une simple révélation de ce qui était déjà en nous "de toute éternité ». Révélations de vérités (de Vérité au singulier ?) non pas seulement prouvées intellectuellement mais, par récurrence en notre vie même, sans cesse éprouvées comme vraies. "Tu ne me chercherais pas si tu ne m'avais (déjà) trouvé » (Pascal).

Rencontre d'un philosophe et d'un peintre qui a permis à l'artiste de voir plus clairement ce qu'il vit profondément, au philosophe peut-être de voir, développée en formes et en couleurs concrètes, une des caractéristiques profondes de sa pensée... sans cesse en quête de Vérité.

Chance d'avoir eu, posé sur nos œuvres, un regard de sympathie, de totale ouverture, une sensibilité extrême servie par une très profonde intelligence !

Cette chance, nous comptons bien ne pas la rendre vaine.

Chabournay, février 2012 